\title{
Outer Disk Star Formation in HI selected Galaxies
}

\author{
G. R. Meurer \\ International Centre for Radio Astronomy Research \\ email: gerhardt.meurer@icrar.org
}

\begin{abstract}
The HI in galaxies often extends past their conventionally defined optical extent. I report results from our team which has been probing low intensity star formation in outer disks using imaging in $\mathrm{H} \alpha$ and ultraviolet. Using a sample of hundreds of $\mathrm{HI}$ selected galaxies, we confirm that outer disk HII regions and extended UV disks are common. Hence outer disks are not dormant but are dimly forming stars. Although the ultraviolet light in galaxies is more centrally concentrated than the HI, the UV/HI ratio (the Star Formation Efficiency) is nearly constant, with a slight dependency on surface brightness. This result is well accounted for in a model where disks maintain a constant stability parameter $\mathrm{Q}$. This model also accounts for how the ISM and star formation are distributed in the bright parts of galaxies, and how HI appears to trace the distribution of dark matter in galaxy outskirts.
\end{abstract}

Keywords. galaxies: structure, galaxies: ISM, galaxies: star formation, galaxies: dwarf, galaxies: spiral, galaxies: irregular

\section{Introduction}

The realization of the importance of the outskirts of galaxies as an environment for disks to evolve and create new stars goes back to the beginning of extragalactic H I studies, 50-60 years ago, when the large extent of galaxies in H I was first noticed (e.g. van de Hulst et al. 1957; Högland \& Roberts 1965; Roberts 1966; Gordon et al. 1968). Here I will emphasize some studies that I found to be particularly inspiring in this field mainly by astronomers attending this meeting.

Albert Bosma's doctoral research (Bosma 1981a,b) showed that at large radii $r$ the observed H I surface density in spiral galaxies traces the projected total mass density as derived from rotation curves. Since this is dominated by Dark Matter (DM) in galaxy outskirts this result has typically been interpreted as H I traces DM, and has been dubbed the "Bosma effect" (e.g. Carignan et al. 1989; Carignan \& Puche 1990a,b; Carignan et al. 1990; Hoekstra et al. 2001, Hessman \& Ziebart 2011). Explanations for it include that DM is gaseous (e.g. Pfenniger et al. 1994; Pfenniger \& Combes 1994) or that there is no DM in galaxy outskirts, but rather the H I disk dominates and that the gravitational force-law is given by Modified Newtonian Dynamics (MOND; Sanders \& McGaugh 2002). Either interpretation presents huge problems for the more accepted paradigm of the Cold Dark Matter (CDM) dominated galaxy evolution. Under CDM dark matter should be dissipationless, whereas a gaseous baryonic DM would be dissipative, while the MOND scenario requires no DM.

Robert Kennicutt (1989) demonstrated that the star formation in spiral galaxies, as traced by $\mathrm{H} \alpha$ emission, often has a sharp edge, beyond which there are few if any $\mathrm{HII}$ regions. He showed that this edge typically corresponds to an increase in the Toomre (1964) stability parameter $Q$. Thus the formation of massive stars is limited to the dynamically unstable portions of galaxies, while the dynamically stable outer disk is 

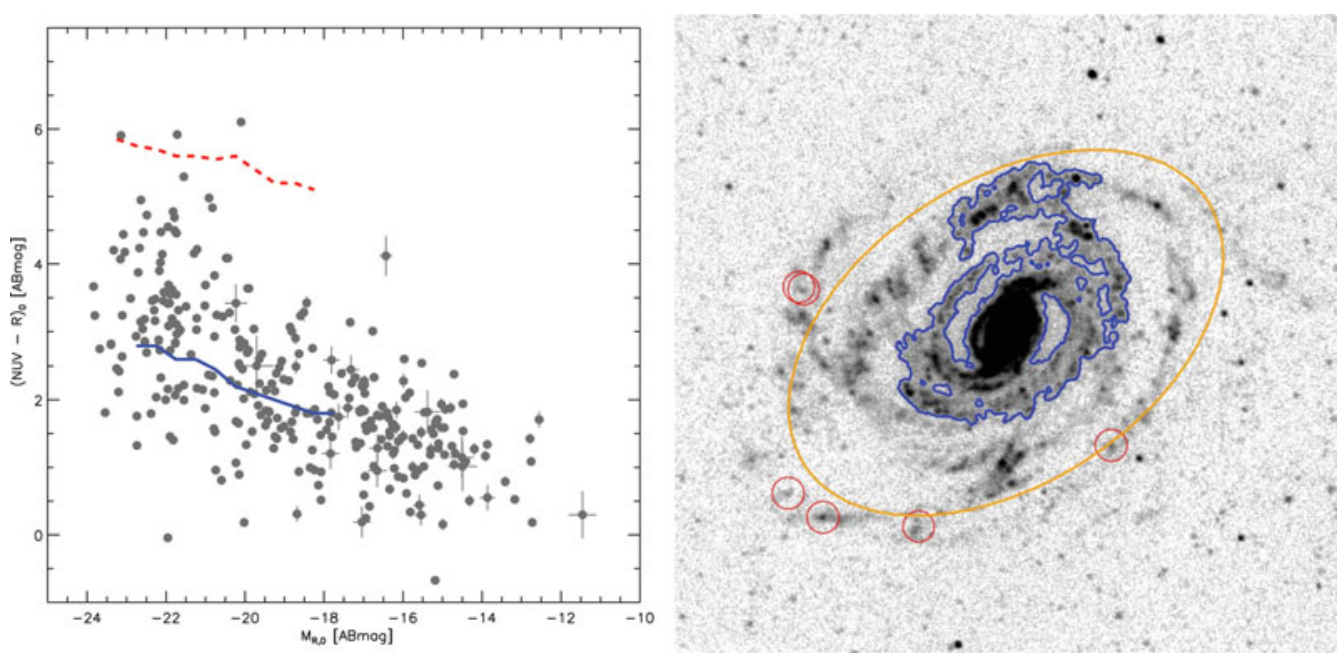

Figure 1. (a) Left: Ultraviolet - optical dust absorption corrected color-magnitude diagram of galaxies in both the SINGG and SUNGG surveys. The dashed and solid lines mark the ridge lines for the passive and star-forming sequences, respectively, of galaxies selected from the SDSS (Wyder et al. 2007). The latter is often known as the "main-sequence of star forming galaxies". Note how the star forming sequence can be traced to much lower luminosities in our surveys and that there are very few HI selected passive sequence galaxies. (b) Right: GALEX NUV image of HIPASS J0052-31 (NGC 289). The irregular contour corresponds to the NUV surface brightness $27.35 \mathrm{ABmag} \operatorname{arcsec}^{-2}$ used to identify XUV1 disks. The extensive UV emission beyond this isophote marks J0052-31 as an XUV1 disk galaxy. The ellipse shows the area beyond which H II regions can be classified as ELdots. Six $\mathrm{H} \alpha$ sources classified as ELdots are circled.

virtually free of star formation. This result was reaffirmed using a larger sample and better data by Martin \& Kennicutt (2001).

Since the work of Kennicutt (1989) it became apparent that outer disks were not totally "dead". The deep H $\alpha$ images of Annette Ferguson (et al. 1998) showed that faint H II regions were common beyond the star formation edge (also shown by Martin \& Kennicutt 2001). With the launch of the GALEX satellite it became clear that outer disks were far from dormant when observed in a different star-formation tracer: ultraviolet (UV) emission. Spectacular examples of Extended Ultraviolet (XUV) disk galaxies were shown in papers lead by Dave Thilker (et al. 2005), and Armando Gil de Paz et al. (2005). The case of M83 is particularly troubling: the distinct edge seen in the $\mathrm{H} \alpha$ radial profiles of Martin \& Kennicutt (2001) were not at all apparent in far UV profiles (Thilker et al. 2005).

Below, I present some results from projects I have been doing primarily with students and postdocs on gas and star formation in the outer disks of galaxies. After describing our star formation surveys in Sec. 2, I summarize our ongoing work on surveying star formation in the outskirts of galaxies, in both $\mathrm{H} \alpha$ and UV emission in Sec. 3. In Sec. 4 I present a model for explaining the distribution of gas and star formation in galaxies, which is particularly relevant for outer disks.

\section{SINGG and SUNGG}

Our work is primarily based on an H I selected sample of galaxies studied in the light of two different star formation tracers. The starting point is the H I Parkes All Sky 


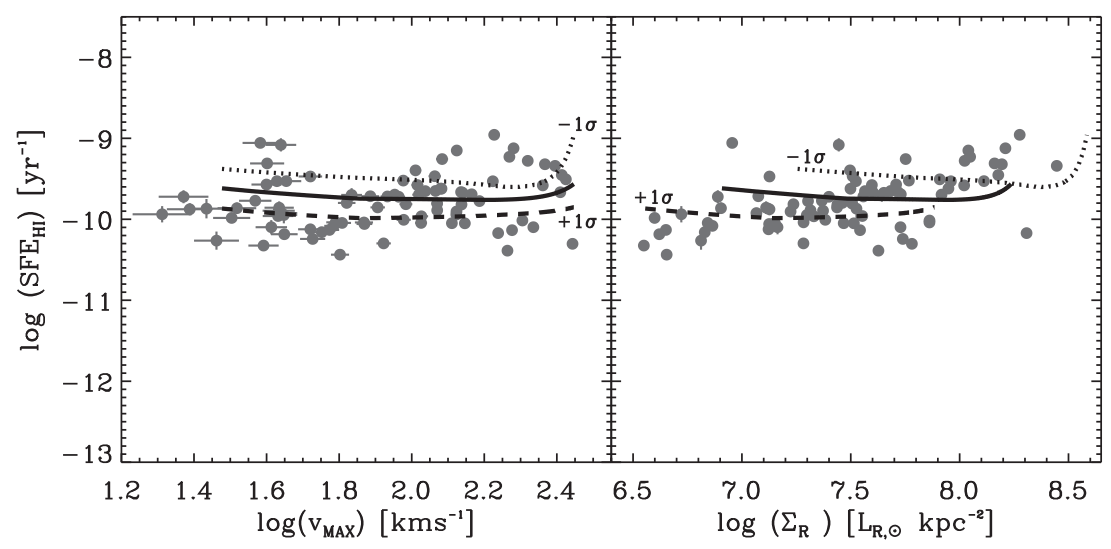

Figure 2. Star Formation Efficiency as a function of circular velocity $V_{\max }$ (left) and effective $R$-band surface brightness (right). Data from SUNGG are shown as circles. Our standard model sequence is shown with the solid line. The dotted (dashed) line show cases where the spatial scale for each model was contracted (expanded) by a factor of 1.4 to simulate a decrease (increase) in angular momentum. For full details see Wong et al. (2016).

Survey (HiPASS Meyer et al. 2004; Koribalski et al. 2004; Wong et al. 2006). HiPASS found $4315 \mathrm{HI}$ sources south of a declination of $\delta=+2^{\circ}$ with the Parkes $64 \mathrm{~m}$ radio telescope. We selected a sample of 468 HiPASS sources to observe in $\mathrm{H} \alpha$ and the $R$ band continuum for the Survey for Ionization in Neutral Gas Galaxies (SINGG) primarily with the CTIO $1.5 \mathrm{~m}$ and $0.9 \mathrm{~m}$ telescopes (Meurer et al. 2006). A total of 331 HIPASS targets were observed, often revealing multiple $\mathrm{H} \alpha$ emitting galaxies. Similarly we selected 139 HIPASS targets from the same parent sample to observe in the far and near UV (FUV and NUV) for the Survey for Ultraviolet emission in Neutral Gass Galaxies (SUNGG; Wong 2007). Using archival data and also analyzing other HIPASS sources that lie in the same GALEX field of view, we ended with observations of 313 HIPASS targets.

No survey is without biases. Ours are biased towards the star forming mains sequence, and away from the red sequence, as shown in Figure 1a. This is expected. Our surveys are meant to target galaxies with an interstellar medium (ISM) and hence those that are capable of forming stars, and then search for new stars in them. Since the red sequence is predominantly free of a cool or cold ISM very few are in our parent sample. A strength of our selection is that we can trace star forming galaxies much further down the star forming main-sequence than traditional flux limited surveys, as shown in the Figure. We are still able to derive properties averaged over large cosmic volumes by tying our results to the H I mass function as done by Hanish et al. (2006) who calculated the $\mathrm{H} \alpha$ based star formation rate density of the local universe from SINGG.

The star formation tracers $\mathrm{H} \alpha$ and FUV are sensitive to $\mathrm{O}$ stars, and both $\mathrm{O}$ and $\mathrm{B}$ stars, respectively. The lower limit to the initial masses of these stars are 18 and 3 $\mathcal{M}_{\odot}$, respectively. Hence, comparing results from these surveys allows us to probe the Initial Mass Function (IMF) of stars, although ones has to be careful to account for other parameters that effect this ratio, specifically star formation history, and dust content. Probing the IMF was one of the major goals of our surveys, and we did so in Meurer et al. (2009). There we showed that the ratio of $\mathrm{H} \alpha$ to FUV fluxes varies systematically with several parameters, most strongly with $R$-band surface brightness. Similar results were found by Janice Lee et al. (2009). After carefully considering other possibilities we argued these results are most easily explained with an IMF deficient in high mass stars in low surface brightness galaxies (Meurer et al. 2009). 


\section{Surveying outer disk star formation}

Surveying with SINGG: ELdots. We searched the SINGG dataset for small line emitting sources projected far from any obvious host. We dubbed Emission Line Dots or ELdots (Ryan-Weber et al. 2004; Werk et al. 2008; Werk et al. 2010a,b; Werk et al. 2011; Santiago-Figueroa et al. 2011). The name perfectly describes their apparent properties, but leaves open their nature. The ambiguity is essential since without additional information it is unclear where these sources are. They may be $\mathrm{H} \alpha$ from an outer H II region, a tiny dwarf galaxy in the immediate vicinity of the H I target, a small galaxy up to several Mpc away (see e.g. Santiago-Figueroa et al. 2011) or a bright background galaxy emitting in [O III], [O II] or some other bright line. While, in spectacular cases many ELdots are arranged in obvious tidal arms indicating most are local to the host, spectroscopy or very high resolution imaging (e.g. HST; Werk et al. 2008) are the only sure ways to determine where the ELdots are relative to the HIPASS target. However, combining UV and $\mathrm{H} \alpha$ photometry allows likely background ELdots to be identified by their relatively red $F U V-R$ color (Werk et al. 2010a).

Systems hosting local ELdots often show signs of interaction or mergers (Ryan-Weber et al. 2004; Werk et al. 2010a). Werk et al. (2011) searched for ELdots around merging systems from the "H I Rogues" sample (Hibbard et al. 2001), and determined their metallicities spectroscopically to test the hypothesis that these outer H II regions may represent the first star formation in chemically pristine ISM from the outskirts of galaxies. However, they found the metallicity gradients are weak and the merger outskirts have uniform modest abundances (typically around half solar). This is also true for the cases of ELdots in systems that are isolated like NGC 2915 (Werk et al. 2010b) which consists of a blue compact dwarf galaxy surrounded by a spectacular H I disk (Meurer et al. $1994 ; 1996)$.

We have identified 219 ELdots in 104 HiPASS targets in the full SINGG dataset; about one third of all the HIPASS fields we surveyed contain at least one ELdot (El Agali et al. 2016 in prep). We have deep GALEX data (exposure times greater than 1000s), for 36 of the fields with ELdots allowing a crude classification of ELdots as local or background. Of the 106 ELdots in those fields, slightly more than half, 58, were found to be local (most likely outer H II regions although see Santiago-Figueroa et al. 2011). A large fraction $(\sim 1 / 3)$ of the hosts appear to be strongly interacting, while nearly half have at least one companion.

Surveying with SUNGG: XUV disk galaxies. Over the austral summer 2015/6 we began a project to classify the SUNGG GALEX images in search of extended UV (XUV) disks using the definitions of Thilker et al. (2007). We concentrated on type 1 XUV disks (XUV1), identified by having obvious signs of star formation beyond an UV isophote designed to be similar to the star formation threshold of Kennicutt (1989). This initial morphological classification was done by summer student Leah Kalimeris, and were checked by Dave Thilker and myself.

We started with 360 HiPASS Targets containing 398 galaxies (i.e. including multiples from SINGG). After rejecting defective images, and other problems, we were left with 332 galaxies to classify. We found $23 \%$ are clearly XUV1; $31 \%$ clearly are not, and $46 \%$ were uncertain. Note these numbers are the preliminary values presented at the meeting. I can confidently say the final fraction of XUV1 galaxies in our sample will be higher than $23 \%$ but will depend on how we draw the line between what is clearly an XUV1 galaxy and what isn't (Kalimeris et al. 2016, in prep.).

In comparison, Thilker et al. (2007) considered a sample of 189 galaxies within 40 Mpc from the GALEX Nearby Galaxy Atlas (Gil de Paz et al. 2007) having optical 
morphologies between S0 and Sm and found $16 \%$ to be XUV1 disks. The higher rate of XUV1 galaxies in our (considerably larger) sample may be due to the H I selection which includes many more dwarf galaxies and avoids gas poor disks.

Overall Impressions. The definitions for ELdots and XUV disks differ in detail but both select star formation beyond the typically defined outer edge of galaxies. With both methods we find $\gtrsim 20 \%$ of H I selected galaxies have outer disk star formation. There are many cases of galaxies with both ELdots and XUV1 disks, an example is shown in Figure 1b. The overall impression I get is that XUV disks are more common than galaxies with ELdots, and similarly that outer disks are more apparent in the UV than $\mathrm{H} \alpha$. This impression hints at the possibility that the IMF in the outer disk is deficient in high mass stars as seen in LSB galaxies (Meurer et al. 2009). This is at apparent odds with the results of Koda et al. (2012) who find that the statistics of the number of UV bright knots in the outer disk of M83 with and without $\mathrm{H} \alpha$ emission as well as the integrated $\mathrm{H} \alpha$ to FUV flux ratio from these knots are consistent with a standard IMF, that is like the bright portion of galaxies. However, in that study only the light in these knots are measured; their method is insensitive to diffusely distributed stars. To square this result with the sharp difference between the integrated $\mathrm{H} \alpha$ and FUV surface brightness profiles of M83 noted in the introduction requires that there be considerable UV bright star formation in lower mass clusters or in individual stars. This is consistent with HST imaging of the outer disks of NGC 2915 (Bruzzese et al. 2015) and M83 (Bruzzese 2016) where the obvious young clusters contain only a small portion of the main-sequence stars. Further work is clearly needed to understand the nature of low intensity star formation in both dwarf galaxies and outer disks.

\section{Disks and Galaxy Evolution}

We are developing a model to explain the range of properties seen along the main sequence of star forming galaxies. Inspired by Leroy et al. (2008), as well as earlier studies (Kennicutt 1989; Meurer et al. 1996; Martin \& Kennicutt 2001), it became clear that disk stability plays an important role in determining disk structure. Hence, the underlying assumption of our model is that galaxy disks evolve to have a uniform stability.

In Meurer et al. (2013) we demonstrated that in the limit of a flat rotation curve, the gas density in a constant $Q$ disk falls off with radius as $r^{-1}$, the same projected mass distribution of the DM halo. This then is the explanation for the Bosma effect - disks equilibrate to trace the dominant mass when the rotation curve is flat. It also accounts for the anti-correlation of the ratio of $\mathrm{H}$ I and total projected mass density with rotational amplitude, a result that neither the gaseous DM hypothesis nor MOND can explain.

In Zheng et al. (2013) we turned our attention to modelling the optically bright parts of galaxies. Here both gas and stars are important to the mass budget of disks so a two fluid version of the $Q$ parameter is required. We used data from the THINGS survey (Leroy et al. 2008) to construct our models, which require the rotation curve and the stellar mass distribution as inputs. From the definition of the two fluid $Q$, we determine the distribution of the ISM disk, which is apportioned in to molecular and neutral phases. A star formation law then allows us to determine the resulting distribution of star formation. We found that our model roughly reproduces the relevant observed profiles but typically do not match them in great detail. The center can be particularly challenging as the steeply rising rotation curves of bulge dominated galaxies require a much larger central gas content and star formation intensity than usually seen.

We next turned our attention to a curious result found by several teams, and verified by us - that the galaxy averaged ratio of FUV emission to $21 \mathrm{~cm} \mathrm{H} \mathrm{I} \mathrm{emission} \mathrm{is} \mathrm{fairly}$ 
constant over a wide range of parameters (Schiminovich et al. 2010; Huang et al. 2012; Wong et al. 2016). This ratio is often referred to as the Star Formation Efficiency, SFE, the ratio of star formation rate to gas content. I use the term SFE for convenience, but one must bear in mind the standard caveats, namely that UV fluxes are highly susceptible to dust absorption, while high mass star formation correlates more strongly with the molecular rather the neutral component of the ISM in the centers of galaxies (e.g. Bigiel et al. 2008). On the other hand, FUV emission is a direct tracer of star formation, unlike $\mathrm{H} \alpha$ and far infrared emission. It is also less prone to uncertainties in the upper end of the IMF than $\mathrm{H} \alpha$ emission (Meurer et al. 2009; Lee et al. 2009). The ISM in galaxy outskirts is dominated by H I while the molecular ISM is notoriously difficult to detect. Meanwhile, UV emission and blue stars correlate well with H I emission there (Cuillandre et al. 2001; de Blok \& Walter, 2003; Bigiel et al. 2010a,b; Bruzzese et al. 2015).

The insensitivity of SFE to galaxy properties is puzzling because star formation in galaxies is much more centrally concentrated than H I emission (e.g. Leroy et al. 2008). Hence, how does the outer H I "know" how to balance the centralized star formation?

In Wong et al. (2016) we account for this relationship using our constant $Q$ model. The driving property of the model - halo mass, is parameterized by the peak orbital velocity $V_{\max }$. The shape of the rotation curve for a given $V_{\max }$ is taken from the parameterization of Persic et al. (1996). We use observed scaling relations to set the stellar mass distribution which we put in a pure exponential disk (Freeman 1970). We adopt the Wang \& Silk (1994) formula for the two fluid $Q$, and find the best results when we apportion the neutral and molecular phases using a recipe based on the disk mid-plane hydrostatic pressure. As noted above, the radial distribution of the outer disk declines as $r^{-1}$, producing an infinite integral if a cutoff is not introduced. Analysis of data from SINGG, SUNGG, and the sample used by Meurer et al. (2013) indicates the outer edge of the disk in the optical, UV and H I are all given by the radius where the orbital time is $\sim 1$ Gyr (Meurer et al. 2016, in prep.). We adopt this cutoff in our model. After adopting a molecular star formation law, we have radial profiles of H I and SFR which we integrate to determine SFE.

Figure 2 shows our model fits the flat SFE relationship with $V_{\max }$ very well. We find a slight correlation between SFE and R band effective surface brightness. We interpret this as due to angular momentum differences between galaxies. This make sense in that after mass, angular momentum of the halo is the second most important parameter in setting the structure of galactic halos and hence the galaxies they contain (Mo, Mao, \& White 1998). To model the effects of a change in the angular momentum we contract or expand the spatial scale of our standard model by a factor of 1.4, the amount required to match the scatter in the luminosity - surface brightness correlation. This produces the offset lines shown in the figure. We see that the offset curves account reasonably well for the scatter in SFE versus $V_{\max }$, and the tilt of the SFE versus $\Sigma_{\star}$ data.

\section{Summary}

H I selected samples of galaxies are ideally suited to understanding the nature of the outer disks of galaxies because gas in galaxies naturally settles into disks, and the H I survives best in the low density low pressure outskirts of disks. Using $\mathrm{H} \alpha$ and UV followup observations of the HIPASS survey we find over $20 \%$ of H I-selected galaxies have star formation in their outskirts as seen by H II regions or Type 1 XUV disks. We developed a model for the distribution of star formation and the gas phases based on the premise that galaxies evolve to have a constant stability parameter $Q$. We showed that this model accounts for (a) the "Bosma effect", whereby H I appears to trace the distribution of Dark 
Matter in the outskirts of galaxies; (b) predicts (albeit crudely) the radial distribution of star-formation and the ISM phases (molecular and neutral) in the optically bright portion of galaxies, and (c) predicts the lack of correlation of the galaxy averaged Star Formation Efficiency (based on H I and UV measurements) with most global properties of galaxies.

Acknowledgments I gratefully acknowledge the students and postdocs who lead much of the work I reported here: Fiona Audcent-Ross, Ahmed Elagali, Leah Kalimeris, Ivy Wong, and Zheng Zheng. I thank Dave Thilker for co-supervision of students and collaborating on many projects on the outer disks of galaxies. I thank the editors for their patience and a suggestion that improved this contribution.

\section{References}

Bigiel, F., Leroy, A., Walter, F., et al. 2008, AJ, 136, 2846

Bigiel, F., Leroy, A., Walter, F., et al. 2010a, AJ, 140, 1194

Bigiel, F., Leroy, A., Seibert, M., et al. 2010b, ApJ, 720, L31

Bosma, A. 1981a, AJ, 86, 1791

Bosma, A. 1981b, AJ, 86, 1825

Bruzzese, S. M., Meurer, G. R., Lagos, C. D. P., et al. 2015, MNRAS, 447, 618

Bruzzese, S. M. 2016, PhD Thesis, University of Western Australia

Carignan C. \& Beaulieu, S. F. 1989, ApJ, 347, 760

Carignan C. \& Puche, D. 1990a, AJ, 100, 394

Carignan C. \& Puche, D. 1990b, AJ, 100, 641

Carignan, C., Charbonneau, P., Boulanger, F., et al. 1990, A\&A, 234, 43

Cuillandre, J.-C., Lequeux, J., Allen, R. J., et al. 2001, ApJ, 554, 190

de Blok, W. J. G. \& Walter, F., 2003 MNRAS, 341, L39

Ferguson, A., Wyse, R., Gallagher, J. et al., 1998, ApJ, 506, L19

Freeman, K. C. 1970, ApJ, 160, 811

Gil de Paz, A., Madore, B. F., Boissier, S., et al. 2005, ApJ, 619, L29

Gil de Paz, A., Boissier, S., Madore, B. F., et al. 2007, ApJS, 173, 185

Gordon, K. J., Remage, N. H., \& Roberts, M. S. 1968, ApJ, 154, 845

Hanish, D. J., Meurer, G. R., Ferguson, H. C., et al. 2006, ApJ, 649, 150

Hessman, F. V. \& Ziebart, M. 2011, A\&A, 532, 121

Hibbard, J. E., van Gorkom, J. H., Rupen, M. P., et al. 2001, ASP Conference Series, 240, 657

Hoekstra, H., van Albada, T. S., \& Sancisi, R. 2001, MNRAS, 323, 453

Höglund, B. \& Roberts, M. S. 1965, ApJ, 142, 1366

Huang, S., Haynes, M. P., Giovanelli, R., et al. 2012, ApJ, 756, 113

Kennicutt, R. C., Jr. 1989, ApJ, 344, 685

Koda, J., Yagi, M., Boissier, S., et al. 2012, ApJ, 749, 20

Koribalski, B. S., Staveley-Smith, L., Kilborn, V. A., et al. 2004, AJ, 128, 16

Lee, J. C., Gil de Paz, A., Tremonti, C., et al. 2009, ApJ, 706, 599

Leroy, A. K., Walter, F., Brinks, E., et al. 2008, AJ, 136, 2782

Martin, C. L. \& Kennicutt, R. C., III, 2001, ApJ, 555, 301

Meurer, G. R., Mackie, G., \& Carignan, C. 1994, AJ, 107, 2021

Meurer, G. R., Carignan, C., Beaulieu, S. F., et al. 1996, AJ, 111, 1551

Meurer, G. R., Hanish, D. J., Ferguson, H. C., et al. 2006, ApJS, 165, 307

Meurer, G. R., Wong, O. I., Kim, J. H., et al. 2009, ApJ, 695, 765

Meurer, G. R., Zheng, Z., \& de Blok, W. J. G. 2013, MNRAS, 429, 2537

Meyer, M. J., Zwaan, M. A., Webster, R. L., et al. 2004, MNRAS, 350, 1195

Mo, H. J., Mao, S., \& White, S. D. M. 1998, MNRAS, 295, 319

Persic, M., Salucci, P., \& Stel, F. 1996, MNRAS, 281, 27

Pfenniger, D. \& Combes, F. 1994, A\& A, 285, 94 
Pfenniger, D., Combes, F., \& Martinet, L. 1994, A\&A, 285, 79

Roberts, M. S. 1966, ApJ, 144, 639

Ryan-Weber, E. V., Meurer, G. R., Freeman, K. C., et al. 2004, AJ, 127, 1431

Sanders, R. H. \& McGaugh, S. S. 2002, ARA\&A, 40, 263

Santiago-Figueroa, N., Putman, M. E., Werk, J., et al. 2011, PASA, 28, 271

Schiminovich, D., Catinella, B., Kauffmann, G., et al. 2010, MNRAS, 408, 919

Thilker, D. A., Bianchi, L., Boissier, S., et al. 2005, ApJ, 619, L79

Thilker, D. A., Bianchi, L., Meurer, G. R., et al. 2007, ApJS, 173, 538

Toomre, A. 1964, ApJ, 139, 1217

van de Hulst, H. C., Raimond, E., \& van Woerden, H. 1957, Bulletin of the Astronomical Institutes of the Netherlands, 14, 1

Wang, B. \& Silk, J. 1994, ApJ, 427, 759

Werk, J. K., Putman, M. E., Meurer, G. R., et al. 2008, ApJ, 678, 888

Werk, J. K., Putman, M. E., Meurer, G. R., et al. 2010a, AJ, 139, 279

Werk, J. K., Putman, M. E., Meurer, G. R., et al. 2010b, ApJ, 715, 656

Werk, J. K., Putman, M. E., Meurer, G. R., et al. 2011, ApJ, 735, 71

Wong, O. I. 2007, PhD Thesis, University of Melbourne

Wong, O. I., Ryan-Weber, E. V., Garcia-Appadoo, D. A., et al. 2006, MNRAS, 371, 1855

Wong, O. I., Meurer, G. R., Zheng, Z., et al. 2016, MNRAS, 460, 1106

Wyder T. K., Martin D. C., Schiminovich D., et al. 2007, ApJS, 173, 293

Zheng, Z., Meurer, G. R., Heckman, T. M., et al. 2013, MNRAS, 434, 3389 